PROCEEDINGS OF THE

AMERICAN MATHEMATICAL SOCIETY

Volume 132, Number 5, Pages 1241-1250

S 0002-9939(03)07350-7

Article electronically published on December 22, 2003

\title{
HOCHSCHILD COHOMOLOGY OF FROBENIUS ALGEBRAS
}

\author{
JORGE A. GUCCIONE AND JUAN J. GUCCIONE
}

(Communicated by Martin Lorenz)

\begin{abstract}
Let $k$ be a field, $A$ a finite-dimensional Frobenius $k$-algebra and $\rho: A \rightarrow A$, the Nakayama automorphism of $A$ with respect to a Frobenius homomorphism $\varphi: A \rightarrow k$. Assume that $\rho$ has finite order $m$ and that $k$ has a primitive $m$-th root of unity $w$. Consider the decomposition $A=A_{0} \oplus$ $\cdots \oplus A_{m-1}$ of $A$, obtained by defining $A_{i}=\left\{a \in A: \rho(a)=w^{i} a\right\}$, and the decomposition $\mathrm{HH}^{*}(A)=\bigoplus_{i=0}^{m-1} \mathrm{HH}_{i}^{*}(A)$ of the Hochschild cohomology of $A$, obtained from the decomposition of $A$. In this paper we prove that $\mathrm{HH}^{*}(A)=\mathrm{HH}_{0}^{*}(A)$ and that if the decomposition of $A$ is strongly $\mathbb{Z} / m \mathbb{Z}$ graded, then $\mathbb{Z} / m \mathbb{Z}$ acts on $\mathrm{HH}^{*}\left(A_{0}\right)$ and $\mathrm{HH}^{*}(A)=\mathrm{HH}_{0}^{*}(A)=\mathrm{HH}^{*}\left(A_{0}\right)^{\mathbb{Z} / m \mathbb{Z}}$.
\end{abstract}

\section{INTRODUCTION}

Let $k$ be a field, $A$ a finite-dimensional $k$-algebra and $D A=\operatorname{Hom}_{k}(A, k)$ be endowed with the usual $A$-bimodule structure. Recall that $A$ is said to be a Frobenius algebra if there exists a linear form $\varphi: A \rightarrow k$, such that the map $A \rightarrow D A$, defined by $x \mapsto x \varphi$ is a left $A$-module isomorphism. This linear form $\varphi: A \rightarrow k$ is called a Frobenius homomorphism. It is well known that this is equivalent to saying that the map $x \mapsto \varphi x$, from $A$ to $D A$, is an isomorphism of right $A$-modules. From this it follows easily that there exists an automorphism $\rho$ of $A$, called the Nakayama automorphism of $A$ with respect to $\varphi$, such that $x \varphi=\varphi \rho(x)$, for all $x \in A$. It is easy to check that a linear form $\widetilde{\varphi}: A \rightarrow k$ is another Frobenius homomorphism if and only if there exists $x \in A$ invertible, such that $\widetilde{\varphi}=x \varphi$. It is also easy to check that the Nakayama automorphism of $A$ with respect to $\widetilde{\varphi}$ is the map given by $a \mapsto \rho(x)^{-1} \rho(a) \rho(x)$.

Let $A$ be a Frobenius $k$-algebra, $\varphi: A \rightarrow k$ a Frobenius homomorphism and $\rho: A \rightarrow A$ the Nakayama automorphism of $A$ with respect to $\varphi$.

Definition 1.1. We say that $\rho$ has order $m \in \mathbb{N}$ and we write ord $\rho=m$ if $\rho^{m}=i d_{A}$ and $\rho^{r} \neq i d_{A}$, for all $r<m$.

Assume that $\rho$ has finite order and that $k$ has a primitive $\operatorname{ord}_{\rho}$-th root of unity $w$. Since the polynomial $X^{\operatorname{ord}_{\rho}}-1$ has distinct roots $w^{i}\left(0 \leq i<\operatorname{ord}_{\rho}\right)$, the algebra $A$ becomes a $\frac{\mathbb{Z}}{\text { ord }_{\rho} \mathbb{Z}}$-graded algebra

$$
A=A_{0} \oplus \cdots \oplus A_{\text {ord }_{\rho}-1}, \quad \text { where } A_{i}=\left\{a \in A: \rho(a)=w^{i} a\right\} .
$$

Received by the editors November 6, 2002.

2000 Mathematics Subject Classification. Primary 16C40; Secondary 16D20.

Supported by UBACYT X193 and CONICET. 
Let $\left(\operatorname{Hom}_{k}\left(A^{\otimes *}, A\right), b^{*}\right)$ be the cochain Hochschild complex of $A$ with coefficients in $A$. For each $0 \leq i<\operatorname{ord}_{\rho}$, we let $\left(\operatorname{Hom}_{k}\left(A^{\otimes *}, A\right)_{i}, b^{*}\right)$ denote the subcomplex of $\left(\operatorname{Hom}_{k}\left(A^{\otimes *}, A\right), b^{*}\right)$, defined by

$$
\operatorname{Hom}_{k}\left(A^{\otimes n}, A\right)_{i}=\bigoplus_{\widetilde{B}_{i, n}} \operatorname{Hom}_{k}\left(A_{u_{1}} \otimes \cdots \otimes A_{u_{n}}, A_{v}\right),
$$

where $\widetilde{B}_{i, n}=\left\{\left(u_{1}, \ldots, u_{n}, v\right)\right.$ such that $\left.v-u_{1}-\cdots-u_{n} \equiv i\left(\bmod \operatorname{ord}_{\rho}\right)\right\}$. The cochain Hochschild complex $\left(\operatorname{Hom}_{k}\left(A^{\otimes^{*}}, A\right), b^{*}\right)$ decomposes as the direct sum

$$
\left(\operatorname{Hom}_{k}\left(A^{\otimes^{*}}, A\right), b^{*}\right)=\bigoplus_{i=0}^{\operatorname{ord}_{\rho}-1}\left(\operatorname{Hom}_{k}\left(A^{\otimes^{*}}, A\right)_{i}, b^{*}\right) .
$$

Thus, the Hochschild cohomology $\mathrm{HH}^{n}(A)$, of $A$ with coefficients in $A$, decomposes as the direct sum

$$
\mathrm{HH}^{n}(A)=\bigoplus_{i=0}^{\operatorname{ord}_{\rho}-1} \mathrm{HH}_{i}^{n}(A),
$$

where $\mathrm{HH}_{i}^{n}(A)=H^{n}\left(\operatorname{Hom}_{k}\left(A^{\otimes^{*}}, A\right)_{i}, b^{*}\right)$.

The aim of this paper is to prove the following results:

Theorem 1.2. Let $A$ be a Frobenius $k$-algebra, $\varphi: A \rightarrow k$ a Frobenius homomorphism and $\rho: A \rightarrow A$ the Nakayama automorphism of $A$ with respect to $\varphi$. If $\rho$ has finite order and $k$ has a primitive $\operatorname{ord}_{\rho}$-th root of unity $w$, then

$$
\mathrm{HH}^{n}(A)=\mathrm{HH}_{0}^{n}(A), \quad \text { for all } n \geq 0 .
$$

Recall that $A=A_{0} \oplus \cdots \oplus A_{\text {ord }_{\rho}-1}$ is said to be strongly $\mathbb{Z} / \operatorname{ord}_{\rho} \mathbb{Z}$-graded if $A_{i} A_{j}=A_{i+j}$, for all $i, j \in\left\{0, \ldots, \operatorname{ord}_{\rho}-1\right\}$, where $i+j$ denotes the sum of $i$ and $j$ in $\mathbb{Z} / \operatorname{ord}_{\rho} \mathbb{Z}$.

Theorem 1.3. Let $A$ be a Frobenius k-algebra, $\varphi: A \rightarrow k$ a Frobenius homomorphism and $\rho: A \rightarrow A$ the Nakayama automorphism of $A$ with respect to $\varphi$. If $\rho$ has finite order, $k$ has a primitive $\operatorname{ord}_{\rho}$-th root of unity $w$ and $A=A_{0} \oplus \cdots \oplus A_{\text {ord }_{\rho}-1}$ is strongly $\mathbb{Z} / \operatorname{ord}_{\rho} \mathbb{Z}$-graded, then

$$
\mathrm{HH}^{n}(A)=\mathrm{HH}^{n}\left(A_{0}\right)^{\mathbb{Z} / \operatorname{ord}_{\rho} \mathbb{Z}}, \text { for all } n \geq 0 .
$$

Corollary 1.4. Assume that the hypotheses of Theorem 1.3 are verified. If the Hochschild cohomology $\mathrm{HH}^{2}\left(A_{0}\right)=0$, then $A$ is rigid.

Remark 1.5. As is well known, every finite-dimensional Hopf algebra $H$ is Frobenius, and being a Frobenius homomorphism, any right integral $\varphi \in H^{*} \backslash\{0\}$. Moreover, by Proposition 3.6 of $[\underline{S}$, the composition inverse of the Nakayama map $\rho$ with respect to $\varphi$ is given by

$$
\rho^{-1}(h)=\alpha\left(h_{(1)}\right) \bar{S}^{2}\left(h_{(2)}\right),
$$

where $\alpha \in H^{*}$ is the modular element of $H^{*}$ and $\bar{S}$ is the composition inverse of $S$ (note that the automorphism of Nakayama considered in $[\underline{S}$ is the composition 
inverse of the one considered by us). Using this formula and that $\alpha \circ S^{2}=\alpha$, it is easy to check that $\rho(h)=\alpha\left(S\left(h_{(1)}\right)\right) S^{2}\left(h_{(2)}\right)$ and, more generally, that

$$
\rho^{l}(h)=\alpha^{* l}\left(S\left(h_{(1)}\right)\right) S^{2 l}\left(h_{(2)}\right),
$$

where $\alpha^{* l}$ denotes the $l$-fold convolution product of $\alpha$. Since $\alpha$ has finite order with respect to the convolution product and, by the Radford formula for $S^{4}$ (see Theorem 3.8 of $[\mathbf{S}]$ ), the antipode $S$ has finite order with respect to the composition, we have that $\rho$ has finite order. So, the above theorems apply to finite-dimensional Hopf algebras.

We think that the decomposition of $H$ associated with $\rho$ can be useful for studying the structure of finite-dimensional Hopf algebras. In this paper we exploit it in a cohomological level. Recently another decomposition of $H$ has been considered, similar to this one, but distinct, namely, the one associated to $S^{2}$ (see [R-S]).

Example 1.6. Let $k$ a field and $N$ a natural number. Assume that $k$ has a primitive $N$-th root of unity $w$. Let $H$ be the Taft algebra of order $N$. That is, $H$ is the algebra generated over $k$ by two elements $g$ and $x$ subject to the relations $g^{N}=1$, $x^{N}=0$ and $x g=w g x$. The Taft algebra $H$ is a Hopf algebra with comultiplication $\Delta$, counity $\epsilon$ and antipode $S$ given by

$$
\begin{array}{ll}
\Delta(g)=g \otimes g, & \Delta(x)=1 \otimes x+x \otimes g, \\
\epsilon(g)=1, & \epsilon(x)=0, \\
S(g)=g^{-1}, & S(x)=-x g^{-1} .
\end{array}
$$

Using that $t=\sum_{j=0}^{N-1} w^{j} g^{j} x^{N-1}$ is a right integral of $H$, it is easy to see that the modular element $\alpha \in H^{*}$ verifies $\alpha(g)=w^{-1}$ and $\alpha(x)=0$. By the remark above, the Nakayama map $\rho: H \rightarrow H$ is given by $\rho(g)=w g$ and $\rho(x)=w^{-1} x$. Hence, $H=H_{0} \oplus \cdots \oplus H_{N-1}$, where

$$
\begin{aligned}
H_{i} & =\left\{a \in H: \rho(a)=w^{-i} a\right\} \\
& =\left\langle x^{i}, x^{i+1} g, \ldots, x^{N-1} g^{N-i-1}, g^{N-i}, x g^{N-i+1}, \ldots, x^{i-1} g^{N-1}\right\rangle .
\end{aligned}
$$

Let $C_{N}=\left\{1, t, \ldots, t^{N-1}\right\}$ be the cyclic group of order $N$. It is easy to see that $C_{N}$ acts on $H_{0}$ via $t \cdot x^{i} g^{i}=w^{i} x^{i} g^{i}$ and that $H$ is isomorphic to the skew product of $H_{0} \# C_{N}$. By Theorem 1.3 .

$$
\mathrm{HH}^{n}(H)=\mathrm{HH}^{n}\left(H_{0}\right)^{C_{N}} \quad \text { for all } n \geq 0,
$$

where the action of $C_{N}$ on $\mathrm{HH}^{n}\left(H_{0}\right)$ is induced by the one of $C_{N}$ on $\operatorname{Hom}_{k}\left(H_{0}^{\otimes n}, H_{0}\right)$, given by

$$
t \cdot \varphi\left(x^{i_{1}} g^{i_{1}} \otimes \cdots \otimes x^{i_{n}} g^{i_{n}}\right)=g^{N-1} \varphi\left(t \cdot x^{i_{1}} g^{i_{1}} \otimes \cdots \otimes t \cdot x^{i_{q}} g^{i_{q}}\right) g .
$$

\section{Proof of Theorems 1.2 and 1.3}

Let $k$ be a field, $A$ a $k$-algebra and $V$ a $k$-module. To begin, we fix some notation:

(1) As in the introduction, we let $D A$ denote $\operatorname{Hom}_{k}(A, k)$, endowed with the usual $A$-bimodule structure.

(2) We let $V^{\otimes n}$ denote the $n$-fold tensor product $V \otimes \cdots \otimes V$. 
(3) Given $x \in A \cup D A$, we write

$$
\pi_{A}(x)=\left\{\begin{array}{ll}
x & \text { if } x \in A, \\
0 & \text { if } x \in D A,
\end{array} \quad \text { and } \quad \pi_{D A}(x)= \begin{cases}x & \text { if } x \in D A, \\
0 & \text { if } x \in A .\end{cases}\right.
$$

(4) For $n \geq 1$, we let $B^{n} \subseteq(A \oplus D A)^{\otimes n}$ denote the vector subspace spanned by $n$-tensors $x_{1} \otimes \cdots \otimes x_{n}$ such that exactly 1 of the $x_{i}$ 's belongs to $D A$, while the other $x_{i}$ 's belong to $A$.

(5) Given $i<j$ and $x_{i}, x_{i+1}, \ldots, x_{j} \in A \cup D A$, we write $\mathbf{x}_{i, j}=x_{i} \otimes \cdots \otimes x_{j}$.

(6) For each map $f: X \rightarrow Y$ and each element $x \in X$, we let $\langle f, x\rangle$ denote the evaluation of $f$ in $x$.

2.1. The complex $X^{*, *}(A)$. For each $k$-algebra $A$, we consider the double complex

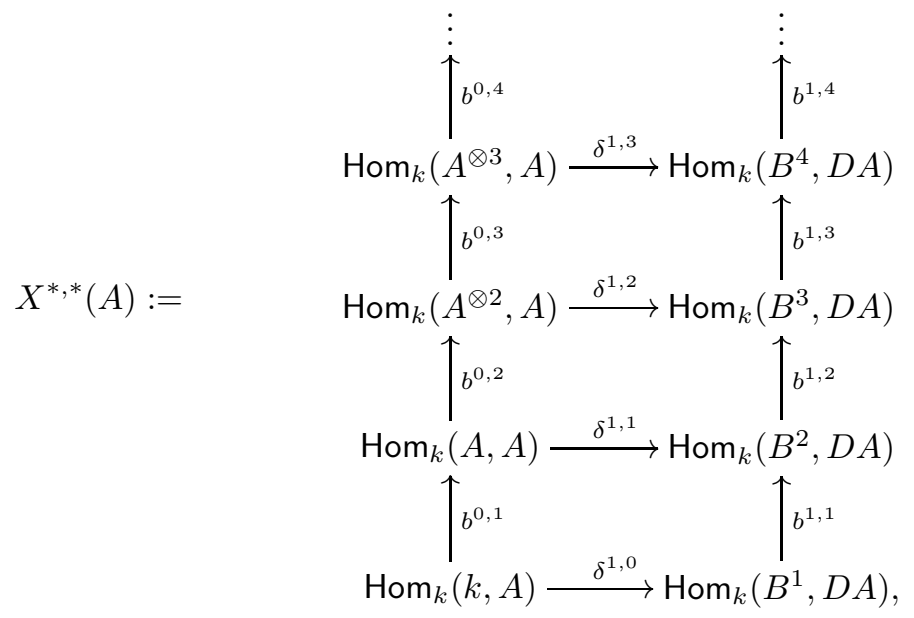

where

$$
\begin{aligned}
\left\langle\left\langle b^{0, n+1}, f\right\rangle, \mathbf{x}_{1, n+1}\right\rangle & =x_{1}\left\langle f, \mathbf{x}_{2, n+1}\right\rangle+\sum_{i=1}^{n}(-1)^{i}\left\langle f, \mathbf{x}_{1, i-1} \otimes x_{i} x_{i+1} \otimes \mathbf{x}_{i+1, n+1}\right\rangle \\
& +(-1)^{n+1}\left\langle f, \mathbf{x}_{1, n}\right\rangle x_{n+1}, \\
\left\langle\left\langle b^{1, n}, g\right\rangle, \mathbf{y}_{1, n+1}\right\rangle= & \left\langle\pi_{A}, y_{1}\right\rangle\left\langle g, \mathbf{y}_{2, n+1}\right\rangle+\sum_{i=1}^{n}(-1)^{i}\left\langle g, \mathbf{y}_{1, i-1} \otimes y_{i} y_{i+1} \otimes \mathbf{y}_{i+1, n+1}\right\rangle \\
+ & (-1)^{n+1}\left\langle g, \mathbf{y}_{1, n}\right\rangle\left\langle\pi_{A}, y_{n+1}\right\rangle, \\
\left\langle\left\langle\delta^{1, n}, f\right\rangle, \mathbf{y}_{1, n+1}\right\rangle= & \left\langle\pi_{D A}, y_{1}\right\rangle\left\langle f, \mathbf{y}_{2, n+1}\right\rangle+(-1)^{n+1}\left\langle f, \mathbf{y}_{1, n}\right\rangle\left\langle\pi_{D A}, y_{n+1}\right\rangle,
\end{aligned}
$$

for $f \in \operatorname{Hom}_{k}\left(A^{\otimes n}, A\right), g \in \operatorname{Hom}_{k}\left(B^{n}, D A\right), \mathbf{x}_{1, n+1}=x_{1} \otimes \cdots \otimes x_{n+1} \in A^{\otimes n+1}$ and $\mathbf{y}_{1, n+1}=y_{1} \otimes \cdots \otimes y_{n+1} \in B^{n}$.

Proposition 2.1. Let $X^{*}(A)$ be the total complex of $X^{*, *}(A)$. It is true that

$$
H^{n}\left(X^{*}(A)\right)= \begin{cases}H^{0}\left(X^{0, *}(A)\right) & \text { if } n=0 \\ H^{n}\left(X^{0, *}(A)\right) \oplus H^{n-1}\left(X^{0, *}(A)\right) & \text { if } n \geq 1\end{cases}
$$

Proof. Let

$$
\delta^{1, *}:\left(\operatorname{Hom}_{k}\left(A^{\otimes *}, A\right),-b^{0, *+1}\right) \rightarrow\left(\operatorname{Hom}_{k}\left(B^{*+1}, D A\right), b^{1, *+1}\right)
$$


be the map defined by

$$
\left\langle\left\langle\delta^{1, n}, f\right\rangle, \mathbf{x}_{1, n+1}\right\rangle=\left\langle\pi_{D A}, x_{1}\right\rangle\left\langle f, \mathbf{x}_{2, n+1}\right\rangle+(-1)^{n+1}\left\langle f, \mathbf{x}_{1, n}\right\rangle\left\langle\pi_{D A}, x_{n+1}\right\rangle .
$$

Since $X^{*}(A)$ is the mapping cone of $\delta^{1, *}$, in order to obtain the result it suffices to check that $\delta^{1, *}$ is null homotopic. Let $\sigma_{*}: \operatorname{Hom}_{k}\left(A^{\otimes *}, A\right) \rightarrow \operatorname{Hom}_{k}\left(B^{*}, D A\right)$ be the family of maps defined by

$$
\left\langle\left\langle\left\langle\sigma_{n}, f\right\rangle, \mathbf{x}_{1, n}\right\rangle, a\right\rangle=(-1)^{j n+1}\left\langle x_{j},\left\langle f, \mathbf{x}_{j+1, n} \otimes a \otimes \mathbf{x}_{1, j-1}\right\rangle\right\rangle \quad \text { if } x_{j} \in D A .
$$

We assert that $\sigma_{*}$ is a homotopy from $\delta^{1, *}$ to 0 . By definition,

$$
\begin{aligned}
\left\langle\left\langle b^{1, n},\left\langle\sigma_{n}, f\right\rangle\right\rangle, \mathbf{x}_{1, n+1}\right\rangle & =\left\langle\pi_{A}, x_{1}\right\rangle\left\langle\left\langle\sigma_{n}, f\right\rangle, \mathbf{x}_{2, n+1}\right\rangle \\
& +\sum_{i=1}^{n}(-1)^{i}\left\langle\left\langle\sigma_{n}, f\right\rangle, \mathbf{x}_{1, i-1} \otimes x_{i} x_{i+1} \otimes \mathbf{x}_{i+2, n+1}\right\rangle \\
& +(-1)^{n+1}\left\langle\left\langle\sigma_{n}, f\right\rangle, \mathbf{x}_{1, n}\right\rangle\left\langle\pi_{A}, x_{n+1}\right\rangle .
\end{aligned}
$$

Hence, if $x_{1} \in D A$, then

$$
\begin{aligned}
\left\langle\left\langle\left\langle b^{1, n},\left\langle\sigma_{n}, f\right\rangle\right\rangle, \mathbf{x}_{1, n+1}\right\rangle, x_{n+2}\right\rangle & =(-1)^{n+2}\left\langle x_{1}, x_{2}\left\langle f, \mathbf{x}_{3, n+2}\right\rangle\right\rangle \\
& -\sum_{i=2}^{n+1}(-1)^{n+i}\left\langle x_{1},\left\langle f, \mathbf{x}_{2, i-1} \otimes x_{i} x_{i+1} \otimes \mathbf{x}_{i+2, n+2}\right\rangle\right\rangle ;
\end{aligned}
$$

if $x_{j} \in D A$ for $1<j \leq n$, then

$$
\begin{aligned}
\left\langle\left\langle\left\langle b^{1, n},\left\langle\sigma_{n}, f\right\rangle\right\rangle, \mathbf{x}_{1, n+1}\right\rangle, x_{0}\right\rangle=(-1)^{(j-1) n+j}\left\langle x_{j},\left\langle f, \mathbf{x}_{j+1, n+1} \otimes \mathbf{x}_{0, j-2}\right\rangle x_{j-1}\right\rangle & \\
& -\sum_{i=0}^{j-2}(-1)^{(j-1) n+i}\left\langle x_{j},\left\langle f, \mathbf{x}_{j+1, n+1} \otimes \mathbf{x}_{0, i-1} \otimes x_{i} x_{i+1} \otimes \mathbf{x}_{i+2, j-1}\right\rangle\right\rangle \\
& -(-1)^{j n+j}\left\langle x_{j}, x_{j+1}\left\langle f, \mathbf{x}_{j+2, n+1} \otimes \mathbf{x}_{0, j-1}\right\rangle\right\rangle \\
& -\sum_{i=j+1}^{n}(-1)^{j n+i}\left\langle x_{j},\left\langle f, \mathbf{x}_{j+1, i-1} \otimes x_{i} x_{i+1} \otimes \mathbf{x}_{i+2, n+1} \otimes \mathbf{x}_{0, j-1}\right\rangle\right\rangle \\
& +(-1)^{j n+n}\left\langle x_{j},\left\langle f, \mathbf{x}_{j+1, n} \otimes x_{n+1} x_{0} \otimes \mathbf{x}_{1, j-1}\right\rangle\right\rangle
\end{aligned}
$$

and if $x_{n+1} \in D A$, then

$$
\begin{aligned}
\left\langle\left\langle\left\langle b^{1, n},\left\langle\sigma_{n}, f\right\rangle\right\rangle, \mathbf{x}_{1, n+1}\right\rangle, x_{0}\right\rangle & =\sum_{i=0}^{n-1}(-1)^{n+i+1}\left\langle x_{n+1},\left\langle f, \mathbf{x}_{0, i-1} \otimes x_{i} x_{i+1} \otimes \mathbf{x}_{i+2, n}\right\rangle\right\rangle \\
& -\left\langle x_{n+1},\left\langle f, \mathbf{x}_{0, n-1}\right\rangle x_{n}\right\rangle .
\end{aligned}
$$

On the other hand, if $x_{1} \in D A$, then

$$
\begin{aligned}
\left\langle\left\langle\left\langle\sigma_{n+1},-\left\langle b^{0, n+1}, f\right\rangle\right\rangle, \mathbf{x}_{1, n+1}\right\rangle\right. & \left., x_{n+2}\right\rangle=(-1)^{n+1} \mathbf{x}_{1}\left\langle\left\langle b^{0, n+1}, f\right\rangle, \mathbf{x}_{2, n+2}\right\rangle \\
& =(-1)^{n+1}\left\langle x_{1}, x_{2}\left\langle f, \mathbf{x}_{3, n+2}\right\rangle\right\rangle+\left\langle x_{1},\left\langle f, \mathbf{x}_{2, n+1}\right\rangle x_{n+2}\right\rangle \\
& +\sum_{i=2}^{n+1}(-1)^{n+i}\left\langle x_{1},\left\langle f, \mathbf{x}_{2, i-1} \otimes x_{i} x_{i+1} \otimes \mathbf{x}_{i+2, n+2}\right\rangle\right\rangle ;
\end{aligned}
$$


if $x_{j} \in D A$ for $1<j \leq n$, then

$$
\begin{aligned}
\left\langle\left\langle\left\langle\sigma_{n+1},\right.\right.\right. & \left.\left.\left.-\left\langle b^{0, n+1}, f\right\rangle\right\rangle, \mathbf{x}_{1, n+1}\right\rangle, x_{0}\right\rangle \\
& =(-1)^{j(n+1)}\left\langle x_{j},\left\langle\left\langle b^{0, n+1}, f\right\rangle, \mathbf{x}_{j+1, n+1} \otimes \mathbf{x}_{0, j-1}\right\rangle\right\rangle \\
& =(-1)^{j(n+1)}\left\langle x_{j}, x_{j+1}\left\langle f, \mathbf{x}_{j+2, n+1} \otimes \mathbf{x}_{0, j-1}\right\rangle\right\rangle \\
& +\sum_{i=j+1}^{n}(-1)^{j(n+1)+i-j}\left\langle x_{j},\left\langle f, \mathbf{x}_{j+1, i-1} \otimes x_{i} x_{i+1} \otimes \mathbf{x}_{i+2, n+1} \otimes \mathbf{x}_{0, j-1}\right\rangle\right\rangle \\
& +(-1)^{j(n+1)+n-j+1}\left\langle x_{j},\left\langle f, \mathbf{x}_{j+1, n} \otimes x_{n+1} x_{0} \otimes \mathbf{x}_{1, j-1}\right\rangle\right\rangle \\
& +\sum_{i=0}^{j-2}(-1)^{j(n+1)+i+n-j}\left\langle x_{j},\left\langle f, \mathbf{x}_{j+1, n+1} \otimes \mathbf{x}_{0, i-1} \otimes x_{i} x_{i+1} \otimes \mathbf{x}_{i+2, j-1}\right\rangle\right\rangle \\
& +(-1)^{j(n+1)+n+1}\left\langle x_{j},\left\langle f, \mathbf{x}_{j+1, n+1} \otimes \mathbf{x}_{0, j-2}\right\rangle x_{j-1}\right\rangle ;
\end{aligned}
$$

and if $x_{n+1} \in D A$, then

$$
\begin{aligned}
\left\langle\left\langle\left\langle\sigma_{n+1},-\left\langle b^{0, n+1}, f\right\rangle\right\rangle, \mathbf{x}_{1, n+1}\right\rangle, x_{0}\right\rangle=(-1)^{n+1}\left\langle x_{n+1},\left\langle\left\langle b^{0, n+1}, f\right\rangle, \mathbf{x}_{0, n}\right\rangle\right\rangle \\
=(-1)^{n+1}\left\langle x_{n+1}, x_{0}\left\langle f, \mathbf{x}_{1, n}\right\rangle\right\rangle+\left\langle x_{n+1},\left\langle f, \mathbf{x}_{0, n-1}\right\rangle x_{n}\right\rangle \\
+\sum_{i=0}^{n-1}(-1)^{n+i}\left\langle x_{n+1},\left\langle f, \mathbf{x}_{0, i-1} \otimes x_{i} x_{i+1} \otimes \mathbf{x}_{i+2, n}\right\rangle\right\rangle .
\end{aligned}
$$

The assertion follows immediately from these equalities.

2.2. The complex $Y^{*, *}(A)$. From now on we fix a Frobenius algebra $A$, a Frobenius homomorphism $\varphi: A \rightarrow k$ of $A$ and we let $\rho$ denote the Nakayama automorphism of $A$ with respect to $\varphi$. Let $A_{\rho}$ be $A$, endowed with the $A$-bimodule structure given by $a \cdot x \cdot b:=\rho(a) x b$. Let $\Theta: D A \rightarrow A_{\rho}$ be the $A$-bimodule isomorphism given by $\Theta(\varphi x)=x$, and let

$$
A_{\rho} \stackrel{\mu}{\longleftarrow} A \otimes A_{\rho} \stackrel{b_{1}^{\prime}}{\longleftarrow} A^{\otimes 2} \otimes A_{\rho} \stackrel{b_{2}^{\prime}}{\longleftarrow} A^{\otimes 3} \otimes A_{\rho} \stackrel{b_{3}^{\prime}}{\longleftarrow} A^{\otimes 4} \otimes A_{\rho} \stackrel{b_{4}^{\prime}}{\longleftarrow} \cdots
$$

be the bar resolution of $A_{\rho}$.

Proposition 2.2. The following assertions hold:

(1) The complex

$$
D A \stackrel{\mu^{\prime}}{\longleftarrow} A \otimes B^{1} \otimes A \stackrel{b_{1}^{\prime \prime}}{\longleftarrow} A \otimes B^{2} \otimes A \stackrel{b_{2}^{\prime \prime}}{\longleftarrow} A \otimes B^{3} \otimes A \stackrel{b_{3}^{\prime \prime}}{\longleftarrow} \cdots,
$$

where $\left\langle\mu^{\prime}, x_{0} \otimes x_{1} \otimes x_{2}\right\rangle=x_{0} x_{1} x_{2}$ and

$$
\begin{aligned}
\left\langle b_{n}^{\prime \prime}, \mathbf{x}_{0, n+2}\right\rangle & =x_{0}\left\langle\pi_{A}, x_{1}\right\rangle \otimes \mathbf{x}_{2, n+2}+\sum_{i=1}^{n}(-1)^{i} \mathbf{x}_{0, i-1} \otimes x_{i} x_{i+1} \otimes \mathbf{x}_{i+2, n+2} \\
& +(-1)^{n+1} \mathbf{x}_{0, n} \otimes\left\langle\pi_{A}, x_{n+1}\right\rangle x_{n+2},
\end{aligned}
$$

is a projective resolution of $D A$.

(2) There is a chain map $\Psi_{*}^{\prime}:\left(A^{\otimes *+1} \otimes A_{\rho}, b_{*}^{\prime}\right) \rightarrow\left(A \otimes B^{*+1} \otimes A, b_{*}^{\prime \prime}\right)$, given by

$$
\left\langle\Psi_{n}^{\prime}, \mathbf{x}_{0, n+1}\right\rangle=\sum_{i=0}^{n}(-1)^{i+n} \mathbf{x}_{0, i} \otimes \varphi \otimes\left\langle\rho, x_{i+1}\right\rangle \otimes \cdots \otimes\left\langle\rho, x_{n}\right\rangle \otimes x_{n+1} .
$$

(3) $\Theta \circ \mu^{\prime} \circ \Psi_{0}^{\prime}=\mu$. 
Proof. Items (2) and (3) follow by a direct computation and item (1) is well known. For instance, the family of maps

$$
\sigma_{0}: D A \rightarrow A \otimes B^{1} \otimes A \quad \text { and } \quad \sigma_{n}: A \otimes B^{n} \otimes A \rightarrow A \otimes B^{n+1} \otimes A(n \geq 1),
$$

given by

$$
\begin{aligned}
& \left\langle\sigma_{0}, x\right\rangle=1 \otimes x \otimes 1, \\
& \left\langle\sigma_{n+1}, \mathbf{x}_{0, n+1}\right\rangle= \begin{cases}1 \otimes \mathbf{x}_{0, n+1}+(-1)^{n+1} \otimes x_{0} x_{1} \otimes \mathbf{x}_{2, n+1} \otimes 1 & \text { if } x_{1} \in D A, \\
1 \otimes \mathbf{x}_{0, n+1} & \text { if } x_{1} \notin D A,\end{cases}
\end{aligned}
$$

where $\mathbf{x}_{0, n+1}=x_{0} \otimes \cdots \otimes x_{n+1} \in A \otimes B^{n} \otimes A$, is a contracting homotopy of the complex of item (1) as a $k$-module complex.

Let $Y^{*, *}(A)$ be the double complex

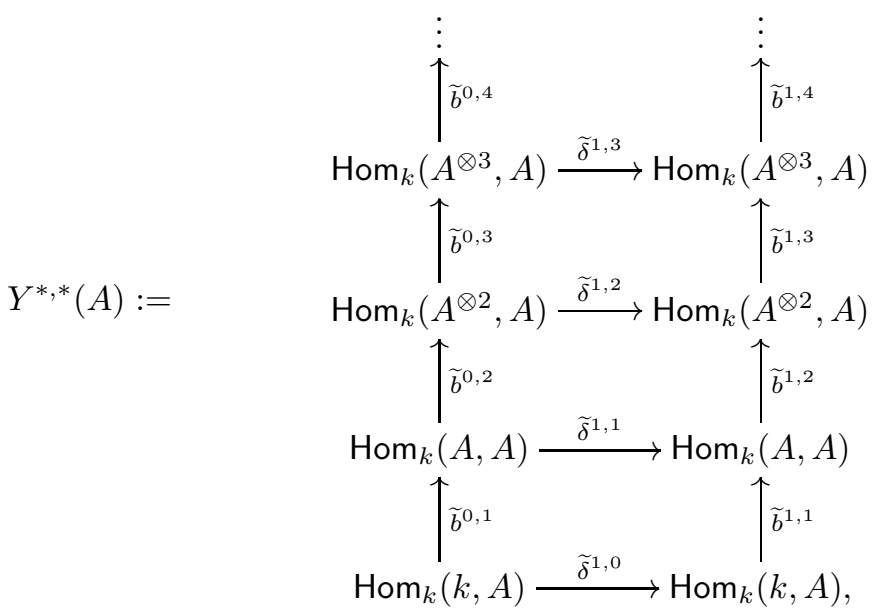

with boundary maps

$$
\begin{aligned}
\left.\left\langle\widetilde{b}^{u, n}, f\right\rangle, \mathbf{x}_{1, n}\right\rangle & =x_{1} f\left(\mathbf{x}_{2, n}\right)+\sum_{i=1}^{n-1}(-1)^{i}\left\langle f, \mathbf{x}_{1, i-1} \otimes x_{i} x_{i+1} \otimes \mathbf{x}_{i+2, n}\right\rangle \\
+ & (-1)^{n}\left\langle f, \mathbf{x}_{1, n-1}\right\rangle x_{n}, \\
\left\langle\left\langle\widetilde{\delta}^{1, n-1}, f\right\rangle, \mathbf{x}_{1, n-1}\right\rangle & =(-1)^{n}\left\langle f, \mathbf{x}_{1, n-1}\right\rangle \\
& +(-1)^{n-1}\left\langle\rho^{-1},\left\langle f,\left\langle\rho, x_{1}\right\rangle \otimes \cdots \otimes\left\langle\rho, x_{n-1}\right\rangle\right\rangle\right\rangle,
\end{aligned}
$$

where $u=0,1, f \in \operatorname{Hom}_{k}\left(A^{\otimes n-1}, A\right)$ and $\mathbf{x}_{1, n}=x_{1} \otimes \cdots \otimes x_{n} \in A^{\otimes n}$.

Proposition 2.3. The double complexes $X^{*, *}(A)$ and $Y^{*, *}(A)$ are quasi-isomorphic.

Proof. It is immediate that $X^{1, *}(A) \simeq \operatorname{Hom}_{A^{e}}\left(\left(A \otimes B^{*+1} \otimes A, b_{*}^{\prime \prime}\right), D A\right)$. Moreover, by Proposition 2.2, the map $\Psi^{*}:=\operatorname{Hom}_{A^{e}}\left(\Psi_{*}^{\prime}, D A\right)$ is a quasi-isomorphism from $\operatorname{Hom}_{A^{e}}\left(\left(A \otimes B^{*+1} \otimes A, b_{*}^{\prime \prime}\right), D A\right)$ to $\operatorname{Hom}_{A^{e}}\left(\left(A^{\otimes *+1} \otimes A_{\rho}, b_{*}^{\prime}\right), D A\right)$. On the other hand, the family of bijective maps

$$
\Upsilon^{n}: Y^{1, n}(A) \rightarrow \operatorname{Hom}_{A^{e}}\left(A^{\otimes n+1} \otimes A_{\rho}, D A\right) \quad(n \geq 0),
$$


defined by $\left\langle\left\langle\Upsilon^{n}, f\right\rangle, \mathbf{x}_{0, n+1}\right\rangle=x_{0}\left\langle f, \mathbf{x}_{1, n}\right\rangle \varphi x_{n+1}$, is an isomorphism of complexes from $Y^{1, *}(A)$ to $\operatorname{Hom}_{A^{e}}\left(\left(A^{\otimes *+1} \otimes A_{\rho}, b_{*}^{\prime}\right), D A\right)$. In fact, we have

$$
\begin{aligned}
\left\langle\left\langle\Upsilon^{n+1},\left\langle\widetilde{b}^{1, n+1}, f\right\rangle\right\rangle, \mathbf{x}_{0, n+2}\right\rangle & =x_{0}\left\langle\left\langle\widetilde{b}^{1, n+1}, f\right\rangle, \mathbf{x}_{1, n+1}\right\rangle \varphi x_{n+2} \\
& =x_{0} x_{1}\left\langle f, \mathbf{x}_{2, n+1}\right\rangle \varphi x_{n+2} \\
& +\sum_{i=1}^{n}(-1)^{i} x_{0}\left\langle f, \mathbf{x}_{1, i-1} \otimes x_{i} x_{i+1} \otimes \mathbf{x}_{i+2, n+1}\right\rangle \varphi x_{n+2} \\
& +(-1)^{n+1} x_{0}\left\langle f, \mathbf{x}_{1, n}\right\rangle x_{n+1} \varphi x_{n+2} \\
& =x_{0} x_{1}\left\langle f, \mathbf{x}_{2, n+1}\right\rangle \varphi x_{n+2} \\
& +\sum_{i=1}^{n}(-1)^{i} x_{0}\left\langle f, \mathbf{x}_{1, i-1} \otimes x_{i} x_{i+1} \otimes \mathbf{x}_{i+2, n+1}\right\rangle \varphi x_{n+2} \\
& +(-1)^{n+1} x_{0}\left\langle f, \mathbf{x}_{1, n}\right\rangle \varphi\left\langle\rho, x_{n+1}\right\rangle x_{n+2} \\
& =\sum_{i=0}^{n}(-1)^{i}\left\langle\left\langle\Upsilon^{n}, f\right\rangle, \mathbf{x}_{0, i-1} \otimes x_{i} x_{i+1} \otimes \mathbf{x}_{i+2, n+2}\right\rangle \\
& +(-1)^{n+1}\left\langle\left\langle\Upsilon^{n}, f\right\rangle, \mathbf{x}_{0, n} \otimes\left\langle\rho, x_{n+1}\right\rangle x_{n+2}\right\rangle \\
& =\left\langle\left\langle\Upsilon^{n}, f\right\rangle,\left\langle b_{n+1}^{\prime}, \mathbf{x}_{0, n+2}\right\rangle\right\rangle .
\end{aligned}
$$

Hence, to finish the proof it suffices to check that $\Upsilon^{*} \circ \widetilde{\delta}^{1, *}=\Psi^{*} \circ \delta^{1, *}$. But,

$$
\begin{aligned}
& \left\langle\left\langle\Psi^{n},\left\langle\delta^{1, n}, f\right\rangle\right\rangle, \mathbf{x}_{0, n+1}\right\rangle \\
& =\sum_{i=0}^{n}(-1)^{i+n} x_{0}\left\langle\left\langle\delta^{1, n}, f\right\rangle, \mathbf{x}_{1, i} \otimes \varphi \otimes\left\langle\rho, x_{i+1}\right\rangle \otimes \cdots \otimes\left\langle\rho, x_{n}\right\rangle\right\rangle x_{n+1} \\
& =(-1)^{n} x_{0} \varphi\left\langle f,\left\langle\rho, x_{1}\right\rangle \otimes \cdots \otimes\left\langle\rho, x_{n}\right\rangle\right\rangle x_{n+1}+(-1)^{n+1} x_{0}\left\langle f, \mathbf{x}_{1, n}\right\rangle \varphi x_{n+1} \\
& =(-1)^{n} x_{0}\left\langle\rho^{-1},\left\langle f,\left\langle\rho, x_{1}\right\rangle \otimes \cdots \otimes\left\langle\rho, x_{n}\right\rangle\right\rangle\right\rangle \varphi x_{n+1}+(-1)^{n+1} x_{0}\left\langle f, \mathbf{x}_{1, n}\right\rangle \varphi x_{n+1} \\
& =x_{0}\left\langle\left\langle\widetilde{\delta}^{1, n}, f\right\rangle, \mathbf{x}_{1, n}\right\rangle \varphi x_{n+1} \\
& =\varphi\left\langle\left\langle\Upsilon^{n},\left\langle\widetilde{\delta}^{1, n}, f\right\rangle\right\rangle, \mathbf{x}_{0, n+1}\right\rangle,
\end{aligned}
$$

as desired.

Proposition 2.4. Let $Y^{*}(A)$ denote the total complex of $Y^{*, *}(A)$. If the Nakayama automorphism $\rho$ has finite order and $k$ has a primitive $\operatorname{ord}_{\rho}$-th root of unity $w$, then

$$
H^{n}\left(Y^{*}(A)\right)= \begin{cases}\mathrm{HH}_{0}^{0}(A) & \text { if } n=0, \\ \mathrm{HH}_{0}^{n}(A) \oplus \mathrm{HH}_{0}^{n-1}(A) & \text { if } n \geq 1 .\end{cases}
$$

Proof. For each $0 \leq i<\operatorname{ord} \rho$, let $Y_{i}^{*, *}(A)$ be the subcomplex of $Y^{*, *}(A)$ defined by

$$
Y_{i}^{u, n}=\bigoplus_{B_{i, n}} \operatorname{Hom}\left(A_{u_{1}} \otimes \cdots \otimes A_{u_{n}}, A_{v}\right)
$$

where $B_{i, n}=\left\{\left(u_{1}, \ldots, u_{n}, v\right)\right.$ such that $\left.v-u_{1}-\cdots-u_{n} \equiv i\left(\bmod \operatorname{ord}_{\rho}\right)\right\}$. It is clear that $Y^{*, *}(A)=\bigoplus_{i=0}^{\text {ord } \rho} Y_{i}^{*, *}(A)$. Let $f \in Y_{i}^{0, n}(A)$. A direct computation shows that

$$
\left\langle\left\langle\widetilde{\delta}_{1, n}, f\right\rangle, \mathbf{x}_{1, n}\right\rangle=(-1)^{n+1}\left(1-w^{-i}\right)\left\langle f, \mathbf{x}_{1, n}\right\rangle .
$$


Hence, the horizontal boundary maps of $Y_{i}^{*, *}(A)$ are isomorphisms if $i \neq 0$, and they are zero maps if $i=0$. So,

$$
H^{n}\left(Y_{i}^{*}(A)\right)= \begin{cases}0 & \text { if } i \neq 0, \\ H^{0}\left(Y_{i}^{0, *}(A)\right) & \text { if } i=0 \text { and } n=0, \\ H^{n}\left(Y_{i}^{0, *}(A)\right) \oplus H^{n-1}\left(Y_{i}^{1, *}(A)\right) & \text { if } i=0 \text { and } n>0,\end{cases}
$$

where $Y_{i}^{*}(A)$ is the total complex of $Y_{i}^{*, *}(A)$. The result follows easily from this fact, since $Y_{0}^{0, *}(A)=Y_{0}^{1, *}(A) \simeq\left(\operatorname{Hom}_{k}\left(A^{\otimes *}, A\right)_{0}, b^{*}\right)$.

Proof of Theorem 1.2, By Proposition 2.3

$$
H^{n}\left(Y^{*}(A)\right)=H^{n}\left(X^{*}(A)\right) \text { and } H^{n}\left(Y^{u, *}(A)\right)=H^{n}\left(X^{u, *}(A)\right) \text {, for } u=0,1 .
$$

Hence, by Propositions 2.1 and 2.4

$$
\begin{aligned}
\mathrm{HH}_{0}^{0}(A) & =H^{0}\left(Y^{*}(A)\right)=H^{0}\left(X^{*}(A)\right) \\
& =H^{0}\left(X^{0, *}(A)\right)=H^{0}\left(Y^{0, *}(A)\right)=\mathrm{HH}^{0}(A)
\end{aligned}
$$

and

$$
\begin{aligned}
\mathrm{HH}_{0}^{n}(A) \oplus \mathrm{HH}_{0}^{n-1}(A) & =H^{n}\left(Y^{*}(A)\right)=H^{n}\left(X^{*}(A)\right) \\
& =H^{n}\left(X^{0, *}(A)\right) \oplus H^{n-1}\left(X^{1, *}(A)\right) \\
& =H^{n}\left(Y^{0, *}(A)\right) \oplus H^{n-1}\left(Y^{1, *}(A)\right) \\
& =\mathrm{HH}^{n}(A) \oplus \mathrm{HH}^{n-1}(A),
\end{aligned}
$$

for all $n \geq 1$. From this it follows easily that $\mathrm{HH}^{n}(A)=\mathrm{HH}_{0}^{n}(A)$, for all $n \geq 0$, as desired.

Proof of Theorem 1.3 . By $[\mathrm{St}]$ or the cohomological version of $\left[\mathrm{L}, \mathbb{Z} / \operatorname{ord}_{\rho} \mathbb{Z}\right.$ acts on $\mathrm{H}^{*}\left(A_{0}, A\right)$ and there is a converging spectral sequence

$$
E_{2}^{p q}=\mathrm{H}^{p}\left(\mathbb{Z} / \operatorname{ord}_{\rho} \mathbb{Z}, \mathrm{H}^{q}\left(A_{0}, A\right)\right) \Rightarrow \mathrm{HH}^{p+q}(A) .
$$

Since $k$ has a primitive $\operatorname{ord}_{\rho}$-th root of unity, $\operatorname{ord}_{\rho}$ is invertible in $k$. Hence, the above spectral sequence gives the isomorphisms

$$
\mathrm{HH}^{n}(A)=\mathrm{H}^{n}\left(A_{0}, A\right)^{\mathbb{Z} / \operatorname{ord}_{\rho} \mathbb{Z}} \quad(n \geq 0)
$$

These maps are induced by the canonical inclusion of $A_{0}$ in $A$, and the action of $i \in \mathbb{Z} / \operatorname{ord}_{\rho} \mathbb{Z}$ on $\mathbf{H}^{n}\left(A_{0}, A\right)$ is induced by the map of complexes

$$
\theta_{i}^{*}\left(\operatorname{Hom}_{k}\left(A_{0}^{\otimes *}, A\right), b^{*}\right) \rightarrow\left(\operatorname{Hom}_{k}\left(A_{0}^{\otimes *}, A\right), b^{*}\right),
$$

defined by

$$
\begin{aligned}
& \left\langle\left\langle\theta_{i}^{n}, \varphi\right\rangle, a_{1} \otimes \cdots \otimes a_{n}\right\rangle \\
& =\sum_{j_{1}, \ldots, j_{n+1} \in J_{i}} s_{i, j_{1}}^{\prime}\left\langle\varphi, s_{i, j_{1}} a_{1} s_{i, j_{2}}^{\prime} \otimes s_{i, j_{2}} a_{2} s_{i, j_{3}}^{\prime} \otimes \cdots \otimes s_{i, j_{n}} a_{n} s_{i, j_{n+1}}^{\prime}\right\rangle s_{i, j_{n+1}},
\end{aligned}
$$

where $\left(s_{i, j}\right)_{j \in J_{i}}$ and $\left(s_{i, j}^{\prime}\right)_{j \in J_{i}}$ are families of elements of $A_{i}$ and $A_{n-i}$, respectively, that satisfy $\sum_{j \in J_{i}} s_{i, j}^{\prime} s_{i, j}=1$. From this it follows easily that we have the isomorphisms

$$
\mathrm{HH}_{i}^{n}(A)=\mathrm{H}^{n}\left(A_{0}, A_{i}\right)^{\mathbb{Z} / \operatorname{ord}_{\rho} \mathbb{Z}} \quad\left(n \geq 0,0 \leq i<\operatorname{ord}_{\rho}\right) .
$$

By combining this result with Theorem [1.2] we obtain the desired result. 


\section{REFERENCES}

[L] M. Lorenz, On the homology of graded algebras, Communications in Algebra, vol. 20 (2) (1992) 489-507. MR 93b:19003

[R-S] R. Radford and H. J. Schneider, On the even powers of the antipode of a finite dimensional Hopf algebra, preprint.

[S] H. J. Schneider, Lectures on Hopf algebras, vol. 31 of Trabajos de Matemática, Facultad de Matemática, Astronomía y Física, Córdoba, 1995. MR 99k:16087

[St] D. Stefan, Hochschild cohomology on Hopf Galois extensions, Journal of Pure and Applied Algebra, vol. 103 (1995) 221-233. MR 96h:16013

Departamento de Matemática, Facultad de Ciencias Exactas y Naturales, Pabellón 1 - Ciudad Universitaria, (1428) Buenos Aires, Argentina

E-mail address: vander@dm.uba.ar

Departamento de Matemática, Facultad de Ciencias Exactas y Naturales, Pabellón 1 - Ciudad Universitaria, (1428) Buenos Aires, Argentina

E-mail address: jjgucci@dm.uba.ar 